\title{
3 Samarkand zwischen Planungsvisionen und Realität
}

\section{Stadtplanung im sowjetischen Samarkand}

$\mathrm{Zu}$ Beginn des 20. Jahrhunderts bestand Samarkand aus zwei voneinander deutlich zu trennenden Hälften - dem historisch gewachsenen Teil, der sogenannten Altstadt, die in der Forschung auch als „timuridische“ oder ,islamische“ Stadt bezeichnet wird, und der seit der russländischen Eroberung in den 1870er-Jahren angelegten Erweiterung nach Südwesten (Abb. 2). ${ }^{103}$ Dem engmaschigen Netz der gewundenen Gassen und traditionellen Hofhäuser aus ungebranntem Lehm wurde eine klare kreis-radiale Struktur gegenübergestellt. Die radialen Straßen laufen auf die Stelle der ehemaligen Zitadelle $\mathrm{zu}$ - diese beiden Teile sind sowohl auf den historischen Plänen als auch auf heutigen Satellitenbildern gut erkennbar. In dieser Kernstruktur manifestiert sich der Dualismus der Stadt, der diese bis heute prägt.

Nach der Oktoberrevolution und der Gründung der Usbekischen SSR hatte Samarkand in den Jahren 1925-1930 den Hauptstadtstatus und erlebte Industrialisierung und Bevölkerungszuwachs ${ }^{104}$ sowie die Gründung diverser Bildungseinrichtungen. ${ }^{105}$ Doch bereits 1930 übernahm Taschkent den Hauptstadttitel und wurde damit zum Hauptschauplatz für städtebauliche Modernisierungsvorhaben in der Usbekischen SSR sowie zum Entscheidungs- und Kompetenzzentrum, von dem aus über die Gestaltung aller anderen Städte der Republik bestimmt wurde. ${ }^{106}$

103 Für eine ausführliche Betrachtung der Bauprozesse in Samarkand nach der Eroberung und bis zu Revolution siehe Nil'sen 1988, S. 93-108.

104 Muminov 1970, S. 103. Die Bevölkerungszahl stieg sprunghaft von 71.000 im Jahre 1923 auf 105.000 im Jahre 1926 an. Als eine der ersten Fabriken wurde 1927 die Seidenspinnerei Hujum eröffnet; unter dem gleichen Namen wie die staatsinitiierte Entschleierungskampagne sollte die Spinnerei symbolisch für die Emanzipation der usbekischen Frauen stehen und zum Flaggschiff der Samarkander Arbeiterklasse werden (Buttino 2015, S. 305-306).

105 Muminov 1970. S. 144-147. Neben diversen Kursen für Alphabetisierung und politische Bildung wurde 1927 die Pädagogische Hochschule gegründet, 1930 das Medizinische und das Landwirtschaftliche Institut.

106 Stronski 2010, S. 31.

Ә Open Access. (C) 2021 Marya Petrova, publiziert von De Gruyter. (c) BY-NC-ND Dieses Werk ist lizenziert unter der Creative Commons Attribution-NonCommercial-NoDerivatives 4.0 International Lizenz. https://doi.org/10.1515/9783110669367-003 


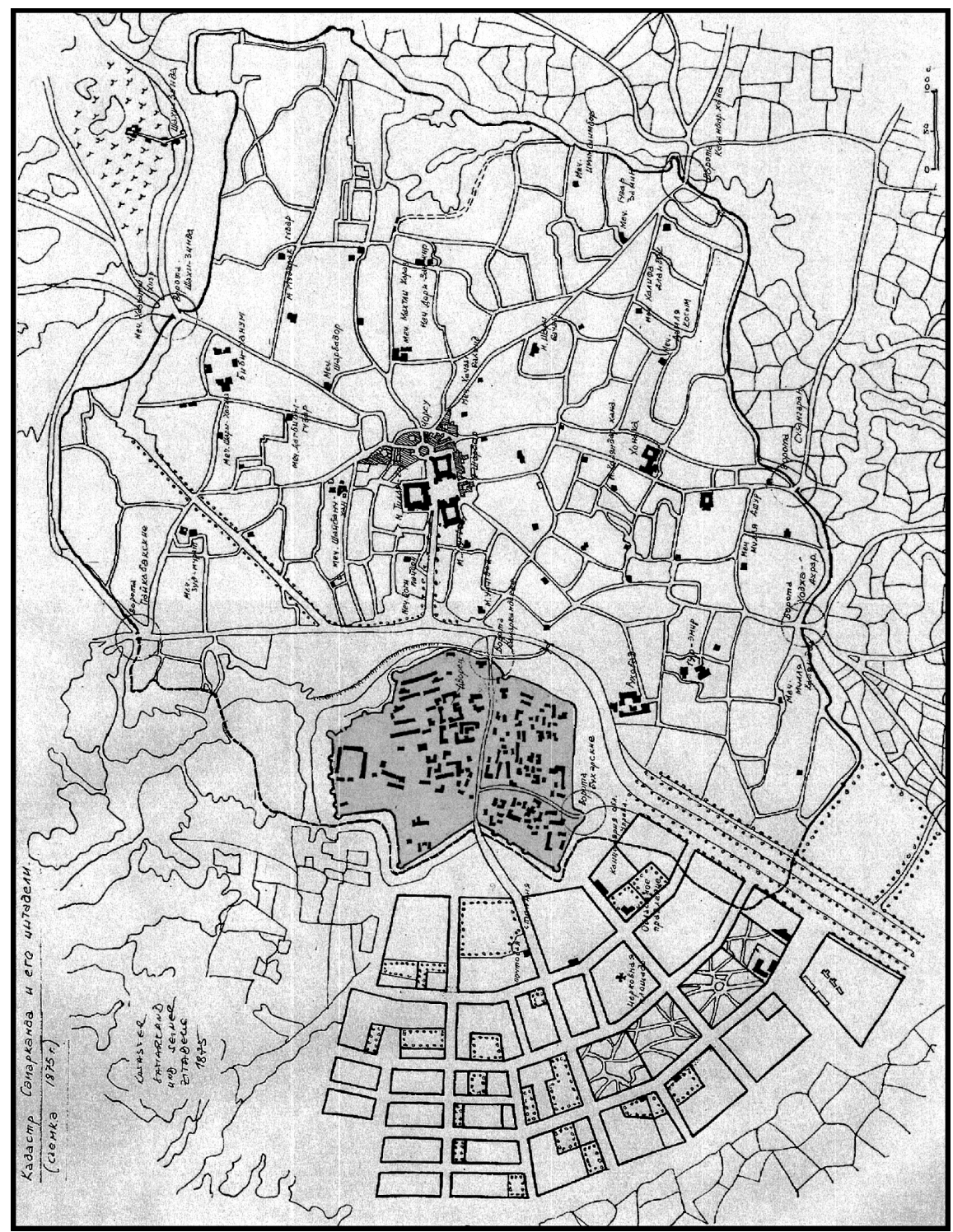

Abb. 2: Katasterbild von Samarkand 1875 mit historischer Altstadt, Zitadelle und bereits angelegter Kolonialstadt. Quelle: Akademie der Künste Taschkent 2004: Gangler et al. 2006, S. 207. 


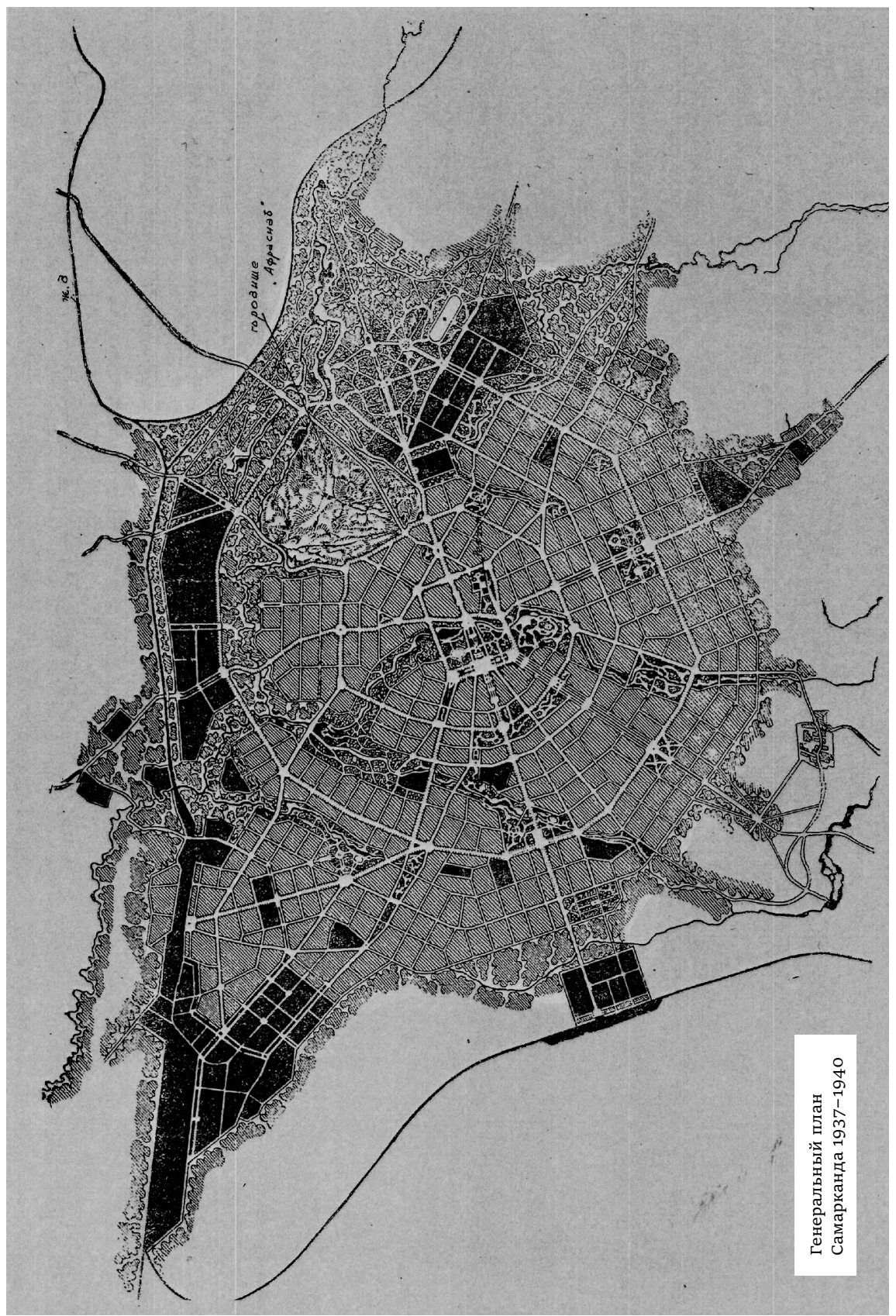

Abb. 3: Generalplan Samarkand 1937-1940. Quelle: Akademie der Künste Taschkent 2004: Gangler et al. 2006, S. 210. 
Das Instrument der Stadtplanung waren die Generalpläne, komplexe Entwürfe mit ganzheitlichem Anspruch für die städtische Entwicklung. Für Samarkand wurde so ein Plan 1937-38 unter der Leitung des Architekten Mitkhat Bulatov $^{107}$ entwickelt. Dabei wurde die vorhandene Struktur der Kolonialstadt als Grundlage genommen und zu einem vollständigen Kreis mit einem gleichmäßigen konzentrisch-radialen Straßennetz mit ebenfalls gleichmäßigen Quartalblöcken ergänzt (Abb. 3). Der Plan war auf eine Gültigkeitsdauer von fünfzehn bis zwanzig Jahren ausgelegt, mit 136.000 Menschen als Zielgröße für die Bevölkerungszahl.

Seine Hauptidee bestand darin, die Zweiteilung von Samarkand in die alte und neue Stadt zu überwinden und ein gemeinsames Zentrum zu schaffen. ${ }^{108}$ Bis auf wenige historische Architekturdenkmäler hätte die Altstadt in dem planmäßig angelegten Raster verschwinden sollen: Die existierenden Straßen sollten erweitert und begradigt werden und an die Stelle von ,engen und staubigen Gassen [sollten] grüne Boulevards“ treten. ${ }^{109}$ Großzügig angelegte Parks und Erholungsgebiete, die das natürliche Stadtrelief in sich integrieren würden, sollten die Landschaft ebenfalls prägen; im Norden bzw. Nordwesten sowie im Südosten wurden Industriegebiete geplant.

In seiner Radikalität und Rücksichtslosigkeit gegenüber vorhandenen Strukturen, insbesondere denen der historischen Altstadt, ist der Plan ein gutes Beispiel für die zeitgenössischen stadtplanerischen Vorstellungen, nämlich das kreis-radiale Planungsprinzip nach dem Vorbild des Moskauer Generalplans ${ }^{110}$ und das Ideal der Stadt als Gesamtensemble, dessen Struktur durchgeplant und Bevölkerungszahl festgelegt werden kann. Es ist nicht ganz klar, wie viel von dem Plan umgesetzt werden konnte, zumal der baldige Ausbruch des Zweiten Weltkriegs ganz andere Aufgaben und Finanzierungsprioritäten in den Vordergrund rückte. Die Rolle, die Samarkand und Zentralasien dabei einnahmen, haben die Entwicklung der Stadt in ganz eigene Bahnen gelenkt.

Im Laufe des Krieges und in den Jahren danach erlebte die Stadt tiefgreifende Veränderungen durch die Verlagerung großer Industriebetriebe und ihrer Mitarbeiter aus dem Nordwesten, die Evakuierung der Menschen aus den Kriegsgebieten sowie Deportation ganzer Volksgruppen wie Tschetschenen und Krimtataren

107 Bulatov war lange Jahre als Stadtplaner und Architekt in Taschkent und in der gesamten Republik tätig (Stronski 2010, S. 61 ff. sowie S. 338 Indexeintrag).

108 Gangler et al. 2006, S. 210. Das gleiche Ziel verfolgten auch unterschiedliche Generalpläne in Taschkent, die ebenfalls das Auflösen des Dualismus der alten „muslimischen“ und der neuen „europäischen“ Stadt zum Ziel hatten. Vgl. dazu Gangler et al. S. 14.

109 Muminov 1970, S. 185.

110 Bohn 2008, S. 5; Gangler et al. 2006, S. 14. 


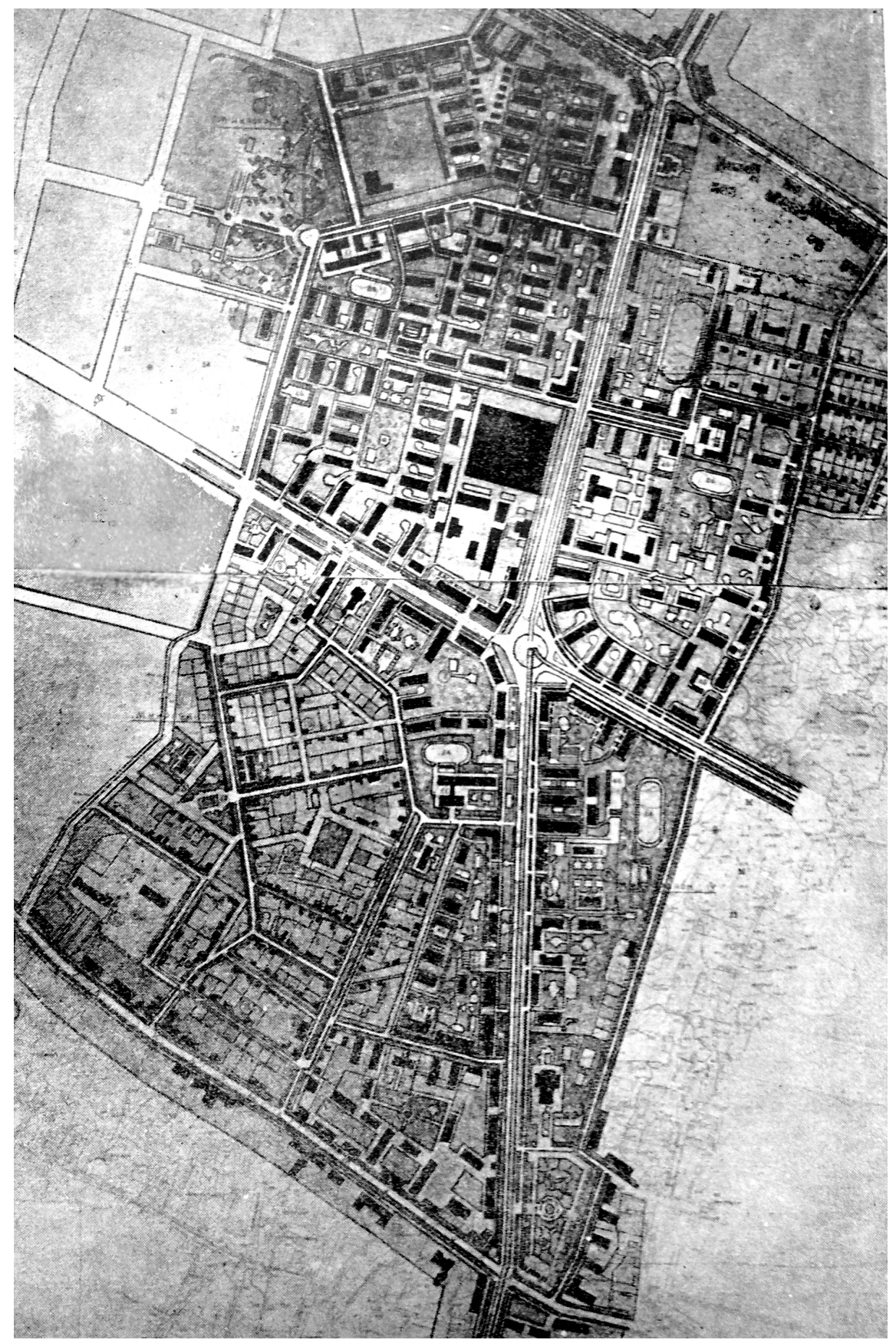

Abb. 4: Detailplan Samarkand für die heutige Gagarina-Straße, um 1960. Babakhanov 1960, S. 9. 
nach Zentralasien, deren Angehörige unter anderem auch in Samarkand landeten. Die evakuierten Betriebe mussten schnell wiederaufgebaut werden und wurden häufig an erstbesten Stellen platziert - dort, wo bereits die nötige Infrastruktur existierte, und ohne Rücksicht auf vorhandene Pläne. Die rapide angestiegene Bevölkerungszahl, Mangel an Wohnraum und das Fehlen von Mitteln, um die Probleme zu lösen, ließen die Wohnsituation und die sanitäre Versorgung in der Stadt lange Zeit dramatisch bleiben. ${ }^{111}$

Ein Jahrzehnt nach Ende des Krieges, zwischen 1954 und 1956, wurde erneut eine Gruppe von Architekten mit der Ausarbeitung bzw. Aktualisierung des alten Generalplans beauftragt. In einer rückblickenden Betrachtung in der Zeitschrift Stroitel'stvo i Arkhitektura Uzbekistana im Jahr 1972 reflektieren die Autoren des Projekts, Tatyana Kalinovskaya und Aleksandr Aleksandrovich, der Plan habe zwar „viele der alten Fehler wiederholt, dabei jedoch der bereits bestehenden Bebauungsstruktur mehr Beachtung geschenkt". ${ }^{112}$ Eine im internen Bericht aus 1962 enthaltene Beschreibung vermittelt ebenfalls den Eindruck, dass viele Ideen und vor allem der Geist des alten Plans, obwohl in weniger radikaler Ausprägung, in der aktualisierten Fassung überlebt haben. ${ }^{113}$ Erkennbar wird dies zum Beispiel am fortdauernden Bestreben, den Dualismus und die Trennung zwischen der Alt- und der neueren Kolonialstadt zu überwinden. Als Nexuspunkt und neues Stadtzentrum wurde hierfür erneut der Platz an der Stelle der ehemaligen Zitadelle gewählt. Das Gelände, wo die von der zaristischen Verwaltung angelegten radialen Straßen zusammenlaufen, wurde für ein weitläufiges Ensemble aus Hotel, Verwaltungs-, Kultur- und Wohnhäusern bestimmt, das in den 1970ern auch tatsächlich gebaut wurde. Die radialen Straßen aus dem 19. Jahrhundert bildeten nach wie vor die Grundlage für die Komposition des Straßennetzes; außerdem ist in dem Dokument die Rede von mehreren kreisförmigen Umfahrungsstraßen, die zum Teil noch wie Schneisen „durchgeschlagen“ werden sollten. Auch das Vorhaben, Industriezonen im Nordwesten und Osten der Stadt zu schaffen, war aus dem Plan von 1938 beibehalten worden. Der Prozess ist so zu verstehen, dass der Generalplan die Bezirke und Quartalblöcke unterteilte und ihre künftige „Füllung“ vorgab, also zum Beispiel festlegte, ob es Viertel mit einstöckigen individuellen oder mit mehrstöckigen Wohnhäusern sein

111 Muminov S. 410. Laut Mumiov hatten im Jahr 1959 lediglich 1,4\% des gesamten Wohnungsbestandes Wasserleitungen; zum Jahr 1969 war diese Zahl auf 30\% gestiegen, was dennoch sehr wenig ist, wenn man bedenkt, dass den Großteil von diesem Anstieg die Geschosswohnungen ausgemacht haben müssen.

112 Kalinovskaya / Aleksandrovich 1972, S. 34.

113 SamOGA, f. 26, op. 1, d.2465, 1. 23-29 
sollten; für die konkrete Ausgestaltung sorgte dann ein sogenanntes proekt detal'noi planirovki „Projekt eines Detailplans“ wie etwa Abb. 4.

Doch bereits ein Jahr nach Fertigstellung des 1954/56er Plans startete das Wohnungsbauprogramm von Khrushchev, das ganz neue Dynamiken in die Bauprozesse auch der Stadt Samarkand brachte. Unter dem Druck der Planvorgaben für neuen Wohnraum mussten große Territorien für die neuen Bezirke, die mikroraions, erschlossen und Parzellen für individuelle Bebauung zugeteilt sowie entsprechende Industrie und Infrastruktur aufgebaut werden. Wie hier noch später gezeigt wird, verliefen diese Prozesse nicht immer nach Plan, denn bereits zu Anfang der 1960er-Jahre berichten die Akten der Architekturbehörde, dass die Umsetzung vieler Vorhaben des Plans durch Verstöße unmöglich gemacht worden war und eine Korrektur erforderlich wurde. ${ }^{114}$ Eine ähnliche Situation herrschte auch in fast allen anderen Städten der Republik: „Die Generalpläne veralten, noch bevor sie geboren werden“, beklagte Architektin Bulycheva, die ebenfalls an der Ausarbeitung des Plans für Samarkand mitwirkte, auf einer Tagung im Jahr 1963. ${ }^{115}$

Kalinovskaya und Alexandrovich formulieren es in einer milderen Form, indem es bei ihnen heißt, dass „die Entwicklung der Stadt zum Jahr 1967 alle Annahmen aus dem Jahr 1956 überholt“ habe, darunter die Bevölkerungszahl, nunmehr 270.000 Personen statt der geplanten 200.000, sowie das Territorium, das zu dem Zeitpunkt bereits die Grenzen erreicht hatte, die eigentlich erst für 1990 anvisiert waren. Aus diesem Grund wurde 1967-68 am zentralen Planungsinstitut in Taschkent (UzGosProekt) ein neuer Generalplan ausgearbeitet und die „technisch-ökonomischen Grundlagen“ für die Stadtentwicklung wurden definiert, zum Teil vom selben Architektenkollektiv. ${ }^{116}$

Der nunmehr dritte Generalpan wurde gleich auf eine Bevölkerungszahl von 500.000 Menschen ausgelegt, und die geplante Baufläche wurde verdoppelt. Die gewachsene radiale Straßenstruktur sollte nach außen hin in eine rechteckige übergehen, in ein Raster aus Magistralen und neuen modernen Wohngebieten, die vor allem in südwestlicher Richtung - bis zum Dargom-Kanal ${ }^{117}$ - gebaut werden sollten (Bild 5c). Parks und grüne Boulevards sollten das Verwaltungszentrum und die alte Innenstadt von den Umfahrungsmagistralen abtrennen. Ein Großteil dieser Pläne sollte erneut nur auf dem Papier bleiben oder in stark abge-

114 SamOGA, f. 1617, op. 1, d. 63, 1. 37.

115 TsGARUz, f. 2532, op. 1, d. 279, 1. 34.

116 Kalinovskaya, Aleksandrovich 1972, S. 34.

117 Für einen Überblick über die Geschichte des Kanals und des Bewässerungssystems in der Samarkander Oase siehe Mantelini 2015. 


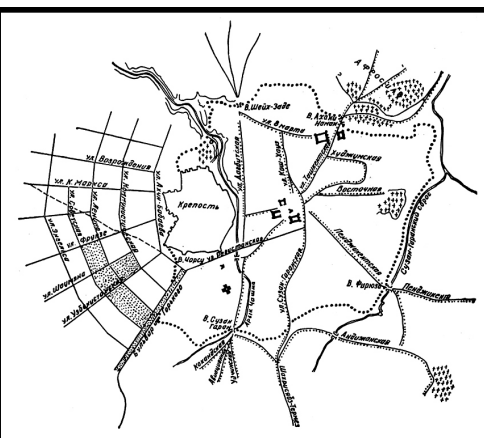

a

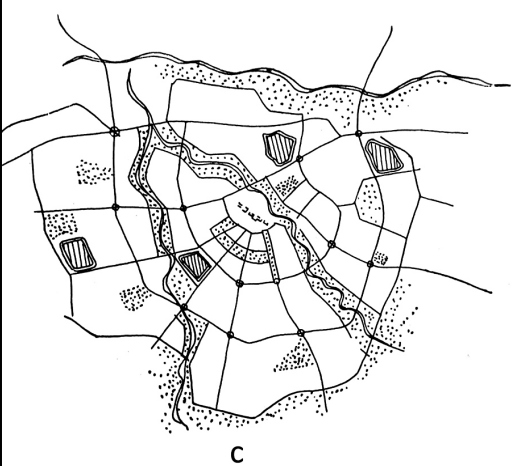

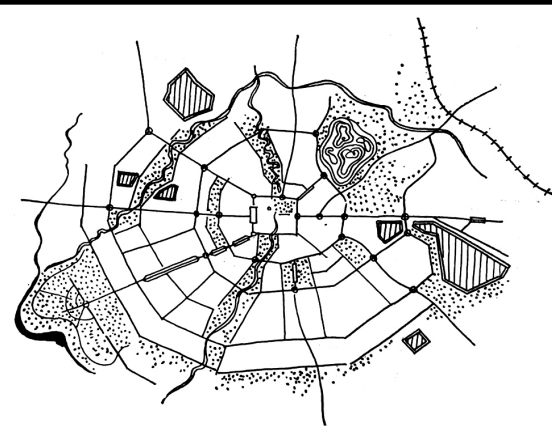

b

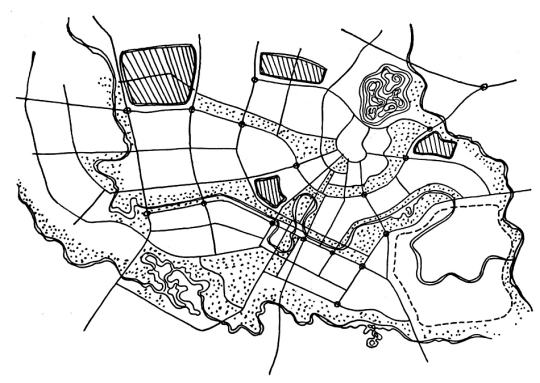

d

Abb. 5: Planungsstadien Samarkand. a - Samarkand Ende 19. Jh.; b - Generalplan 1937-1940; c - Generalplan 1956; d - Generalplan 1967. Kalinovskaya, Aleksandrovich 1972, S. 33-35.

wandelter Form, und manchmal erst Jahrzehnte später, umgesetzt werden. ${ }^{118} \mathrm{Neu}$ in dieser Version ist jedoch der Anspruch, die bestehenden Strukturen, vor allem die der Altstadt, zu berücksichtigen. Laut Plan sollte diese weitgehend von Verkehr und intensiven Bauaktivitäten befreit werden, die vorhandene Struktur der Straßen sowie der lokale Typ der Bebauung mit ein- und zweistöckigen Häusern sollte erhalten bleiben.

Diesen Überlegungen ging eine Fachdiskussion über Rekonstruktion und Umbau der historischen Städte Usbekistans voraus, welche ihrerseits Teil der

118 Die zugängliche Information über die Generalpläne beschränkt sich meist auf allgemeine Beschreibungen, während Dokumente mit detaillierteren Plänen der Geheimhaltung unterliegen. Das macht es schwierig, die genauen Zeitpunkte von Veränderungen zu rekonstruieren. Dank der Funktion „Historische Bilder“ in Google Earth ist es möglich, Veränderungen im Stadtbild seit 1984 (grob) bzw. 2004 (genau) nachzuverfolgen. So wurden zum Beispiel einige Umfahrungsstraßen erst 2017 ,durchgeschlagen“. 
größeren internationalen wie sowjetischen Debatten über das kulturelle Erbe und dessen Erhalt war. ${ }^{119}$ Gleichzeitig dürfte die Entdeckung des Tourismus als wichtige wirtschaftliche und propagandistische Ressource eine Rolle gespielt haben - ab Mitte der 1960er-Jahre kann dies auf den Seiten der Zeitschrift Stroitel'stvo $i$ arkhitektura Uzbekistana nachverfolgt werden. ${ }^{120}$ Die Diskussion, so scheint es, entwickelte sich vor allem zwischen zwei Lagern bzw. fachlichen Bereichen - auf der einen Seite standen die Architekten und Kunsthistoriker, die sich für den Erhalt und eine größere Wertschätzung der lokalen Bauformen und Traditionen und damit auch für die Schonung der historischen Stadtstrukturen einsetzten, auf der anderen die Stadtplaner und Ökonomen mit einer Modernisierungsagenda und dem Anspruch, eine Stadt zu gestalten, deren Infrastruktur und Stadtbild den geltenden Maßstäben einer nicht näher definierten Modernität entsprechen sollten. Die insgesamt zu geringe Dichte der Bevölkerung und die Dominanz der ein- bis zweistöckigen Bebauung in den Altstädten Usbekistans sowie in den neueren Bezirken vieler Städte wurde als problematisch für die perspektivische Entwicklung der Städte gesehen. ${ }^{121}$ Dazu kam das schnelle und zum Teil schlecht kontrollierbare Wachstum der Stadtgebiete, das im Falle der usbekischen Oasenstädte wertvolle landwirtschaftliche Flächen verschwinden ließ und die infrastrukturelle Versorgung in den Städten verteuerte, weswegen viele Architekten mehr Abriss und Verdichtung in den Innenstädten anstelle von Expansion in die Fläche forderten.

Zurück zu der Beschreibung des Generalplans von Samarkand wird deutlich, dass sich in diesem Fall die Erkenntnis oder besser die Einsicht durchgesetzt hatte, dass Architekturdenkmäler nicht losgelöst von den historisch gewachsenen Strukturen der Altstädte mit traditionellen Hofhäusern, Straßen und kleineren Quartalsmoscheen betrachtet werden können und dass diese Elemente ebenso wertvolle Bestandteile des architektonischen und historischen Erbes sind. Gleichzeitig aber wurde ihnen nur eine sekundäre Rolle, nämlich die der Kulisse für eine vorteilhafte Inszenierung der „Hauptdenkmäler“, zugeteilt. Die

119 Zur Entwicklung der internationalen Kooperation im Bereich von Denkmalschutz und Kulturerbe in der UdSSR siehe Geering 2019, zu den Prozessen im sowjetischen Usbekistan siehe Demchenko 2011.

120 Für die Diskussion um den Erhalt und Rekonstruktion in der Zeitschrift Stroitel'stvo i arkhitektura Uzbekistana siehe z.B.: Kryukov / Notkin 1966; Gordeeva 1969; Alexandrovich / Kalinovskaya 1968; Kalinovskaya / Alexandrovich 1972; Notkin / Gordeeva, 1972; Pugachenkova 1973. 121 Gordeeva 1969, S. 24. Der Anteil der ein- bis zweistöckigen Bebauung in den Städten der UzSSR betrug in den 1970er-Jahren 65 bis $90 \%$. 
eigentliche Bausubstanz - mit Mauern aus in der Regel ungebrannten Lehmziegeln oder nach chūbkori-Technik ${ }^{122}$ - wurde als primitiv, marode und nicht mehr den modernen Standards für Hygiene entsprechend bezeichnet. Den Vorstellungen der Stadtplaner zufolge sollten die alten Wohnhäuser zerstört und an ihrer Stelle neue Gebäude errichtet werden, welche aber mit ihren Fassaden die als traditionell deklarierten Formen nachahmen würden. ${ }^{123}$

Konkrete Ideen und Empfehlungen für den Umbau der Altstadt sollten ein Wettbewerb (1970) und ein Forschungsprojekt des Taschkenter Instituts für experimentelle Planung (1972) erbringen. ${ }^{124}$ Die Ergebnisse waren - symptomatisch - erst ein paar Jahre nach dem Generalplan fertig, obwohl die andere Reihenfolge wohl logischer gewesen wäre; außerdem hatten die Empfehlungen keine bindende Wirkung. In der Studie wurde eine Klassifikation der einzelnen Altstadtviertel aufgestellt mit dazugehörigen Moscheen und einzelnen Wohnhäusern, die als erhaltenswert eingestuft wurden. Die gesamte Altstadt sollte demnach in unterschiedliche Bereiche aufgeteilt werden: in ein architektonisches „Schutzgebiet“ (zapovednik) mit touristischer Zone, wo nur archäologische oder restauratorische Eingriffe erlaubt sein würden, und eine Zone freieren Bauens mit neuen und modernen Häusern.

Bezeichnend und charakteristisch für diese Pläne ist die völlige Abwesenheit von Menschen und ihren Bedürfnissen und Interessen - das Fehlen der eigentlichen Bewohner der Stadt also. Sie scheinen nicht existent zu sein, obwohl die modernen Standards implizit der Verbesserung genau ihres Lebens dienen sollten. Doch die Idee von Stadt als sozialem Lebensraum scheint für die sowjetischen Stadtplaner kaum eine Rolle gespielt zu haben; ja nicht einmal eine so grundlegende und technische Frage wie die der Umsiedlung, die unweigerlich mit den Modernisierungsmaßnahmen einhergehen würde, findet in den Diskussionen zur Umgestaltung von Samarkand Erwähnung. Menschen scheinen willenlose Objekte zu sein, die wie Figuren auf einem Spielbrett beliebig verschoben werden können.

Die einzige Architektin (zumindest auf den Seiten der Zeitschrift Stroitel'stvo i Arkhitektura Uzbekistana), die die Bedürfnisse der Einwohner thematisierte, war

122 Eine Technik, die am ehesten mit dem deutschen Fachwerk verglichen werden kann, bei der ein Holzgerüst mit Lehmziegeln ausgefüllt wird.

123 Kryukov / Notkin 1966, S. 28-29.

124 Für die Ergebnisse des Architekturwettbewerbs siehe Shakirov 1971; die Studie wurde vom Taschkenter Institut für experimentelle Planung TashZNIIEP (Tashkentskij Zonal'nyi NauchnoIssledovatel'skij Institut Eksperimental'nogo Proektirovaniya) durchgeführt. Siehe dazu: Grinevich et al. 1972. 
Zlata Chebotareva. Bei der Vorstellung des Experimentalprojekts „Mahalla“ („Stadtviertel“) zur Umgestaltung der Taschkenter Altstadt plädierte sie 1968 für einen Umbau des Viertels in Phasen, sodass die ursprünglichen Bewohner innerhalb des Viertels umgesiedelt werden könnten, da diese ihre „nachbarschaftliche Kontakte schätzen“ und „nicht zu Migration neigen“ würden. ${ }^{125}$ In diesem Vorhaben präsentierte Chebotareva Ideen für standardisierte ein- bis zweistöckige Bebauung, die jeder Familie ein Haus mit einem kleinem Hof ermöglichen würde, wo aber durch kompakte Anordnung die gleiche Bevölkerungsdichte wie bei den mehrstöckigen Häusern erreicht werden könnte. Das Projekt sowie die Empfehlungen wurden jedoch aus verschiedensten Gründen nicht umgesetzt. Das Beispiel macht deutlich, dass, obwohl Architekten und Planern offenbar die Bedürfnisse von Menschen durchaus bekannt waren, ${ }^{126}$ diese entweder keinen großen Stellenwert hatten oder es an politischem und ideologischem Willen fehlte, die Produktionsketten der großen Baukombinate entsprechend umzustellen.

Die Nichtbeachtung der Interessen der Bürger war nur eines der Merkmale des sowjetischen städtebaulichen Denkens. Dieses hatte ganz eigene Logiken, welche die Prozesse und, als Konsequenz, die gebaute Substanz in zahlreichen sowjetischen Städten prägten. So hatte z.B. ein Generalplan (Genplan) als Instrument der Stadtgestaltung eine Doppelrolle: Er war einerseits eine Vision im genuin modernistischen Sinne, die Stadt in Einklang mit geltenden wissenschaftlichen Vorstellungen von Städtebau zu bringen. Damit einher ging der Anspruch, den gegebenen Zustand, den man unbefriedigend fand, zu überwinden - ein herbeigewünschter Qualitätssprung, ein Griff nach dem Utopischen gewissermaßen. Andererseits sollte so ein Genplan eine konkrete Planungsgrundlage für alle Bauvorhaben in der Stadt sein. Die idealisierten Pläne waren meist auf eine entfernte Zukunft ausgerichtet, es fehlten aber häufig Vorstellungen über die Schrittfolge der Umsetzung - was man heute als „Roadmap“ bezeichnen würde; erschwert wurde die Situation durch den Mangel an Ressourcen und ihre ungleiche Verteilung. Diese Beobachtung formuliert Yulia Kosenkova, die mehrere andere Prob-

125 Chebotareva 1968, S. 11.

126 In der zweiten Hälfte der 1960er und bis Anfang/Mitte der 70er, parallel zur Diskussion um den Denkmalschutz, finden sich auf den Seiten der Zeitschrift Stroitel'stvo i Arkhitektura Uzbekistana einige Beiträge, die sich mit den Lebensbedingungen in verschiedenen Wohnformen beschäftigen - von traditioneller mahalla bis hin zu Neubauten in mikroraions (Kontorer 1971), und auch mit klimabedingten Anforderungen an die Neubauten (Sukhanov 1972). Darüber hinaus wurden auch demografische Strukturen untersucht und mit dem neu gebauten staatlichen Wohnraum abgeglichen (Rusanova 1968). 
lemstellen des städtebaulichen Denkens in der Sowjetunion in 13 Hypothesen zusammenfasst. ${ }^{127}$ Sie beobachtet weiter zum Beispiel, dass Stadtplaner in der Regel nur über fragmentarisches Wissen zu den Veränderungen in den Städten verfügten und wenig Methoden zu deren Beobachtung hatten. Auf der Suche nach einem universell „richtigen“ Stadtmodell wurde die Stadt - so Kosenkova - als ein „konfliktloses Objekt betrachtet, das sich einem einzigen Projektwillen unterordnet, während inhärente und willkürliche Mechanismen der Wandelbarkeit nicht beachtet wurden“. ${ }^{128}$

Eine sehr konkrete Gestalt nahmen Pläne und Bauvorhaben im Vorfeld von runden Daten oder Jubiläen an, so zum Beispiel zum 2500-Jahr-Jubiläum von Samarkand, das 1970 gefeiert wurde. Unter dem Druck, die Stadt aufzuräumen und aufzuhübschen, wurden zusätzliche Mittel freigegeben und Kräfte mobilisiert. Die umfangreichen und sehr detailliert aufgelisteten Anweisungen zu Bau und Renovierung, die gefühlt alle kommunal- wie wohnwirtschaftlichen Bereiche der Stadt betreffen, vor allem aber repräsentative Objekte wie Museen, Theater, Restaurants und auch die Straßeninfrastruktur, zeugen von der Bedeutung solcher Anlässe auf der symbolisch-politischen wie auch der praktischen Ebene für die Gestaltung - nicht nur von Samarkand, sondern das gleiche gilt für andere Städte Usbekistans. ${ }^{129}$

Der letzte noch zur Sowjetzeit entworfene Generalplan wurde in den Jahren 1975-1981 ausgearbeitet. In Wirklichkeit scheint er eine Aktualisierung des vorherigen Plans gewesen zu sein mit dem Unterschied, dass die geplante Einwohnerzahl auf 750.000 Einwohner bis 2010 angehoben wurde. Der Plan sah eine Erweiterung nach Süden vor in Verlängerung der Achse des Universitätsboulevards. ${ }^{130}$ Dort wurde ein Wohngebiet für 100.000 Einwohner mit begleitender Infrastruktur und Naherholungsgebieten geplant. Wichtig war zudem der Ausbau der Umfahrungsstraßen mit dem Ziel, den historischen Stadtkern vom Verkehr zu entlasten. Auch diese ambitionierten Vorhaben blieben auf dem Papier: Die wirtschaftliche Krise in der UdSSR, die Kürzung der öffentlichen Ausgaben, die Perestroika und letztlich der Zerfall der UdSSR haben die Umsetzung unmöglich gemacht. $^{131}$

127 Kosenkova 'Gradostroitel'noe myshlenie'. http://www.alyoshin.ru/Files/publika/ kosenkova/kosenkova_mish.html (letzter Zugriff: 07.02.2020).

128 Kosenkova, 'Gradostroitel'noe myshlenie'.

129 SamOGA, f. 1617, op. 1, d. 233, 1. 87-121 sowie SamOGA f. 26, op. 1, d. 3069, 1. 3-12.

130 AKTC 1996, S. 22.

131 Gangler et al. 2006, S. 212. 
Die verschiedenen Etappen in der Stadtplanung zeugen davon, dass es durchaus ernsthafte Ambitionen gab, Samarkand im Einklang mit den zum jeweiligen Zeitpunkt geltenden Vorstellungen von einer modernen sowjetischen Stadt zu bringen. Doch waren die Pläne und ihre Verfasser mit vielen Herausforderungen und Hindernissen konfrontiert, welche die Realisierung erschwerten oder gar unmöglich machten. Die geringen infrastrukturellen wie finanziellen Möglichkeiten der Stadt, die existierende Wohnungsnot sowie Bürger und Bürgerinnen, die ihre Wohnsituation selbst zu lösen versuchten - all das schuf Eigendynamiken, die von den steifen Plänen nur wenig berücksichtigt wurden. Nach der Perspektive vom Reißbrett aus versetze ich nun den Fokus auf die reelle Lage der Stadt.

\section{Die Wohnsituation im Samarkand der 1950er- und 60er-Jahre}

\section{Bevölkerungszahlen und Wohnraummangel}

Die Wohnungsnot, unter der die Sowjetbürger Jahrzehnte nach dem Krieg litten, herrschte sowohl in den durch Kämpfe zerstörten Gegenden als auch im Hinterland, das als Evakuierungsgebiet Millionen von Menschen beherbergen musste. Auch Samarkand war in dieser Hinsicht mit betroffen.

Als erster Ansatzpunkt für ein Verständnis der Wohn- und Bevölkerungssituation können diverse statistische Daten aus Volks- und Wohnraumbestandszählungen dienen. Es ist jedoch nicht ganz einfach, genaue Bevölkerungszahlen für Samarkand zu bekommen, weil die zu findenden Angaben nicht konsistent bzw. nicht vollständig sind und deutliche Unstimmigkeiten zwischen den Quellen auffallen: So variieren die Angaben für das Jahr 1939 von 134.000 bis 191.000 Einwohnern. ${ }^{132} \mathrm{Zu}$ den Evakuierten, die in den Kriegsjahren in die oblast' von Samarkand aufgenommen wurden, existiert eine Zahl von 165.000. ${ }^{133}$ Ein Bericht über den Wohnraumbestand aus dem Jahr 1960 schätzt die Einwohnerzahl auf

132 Bei Muminov finden wir die Zahl 151.000 im Jahre 1937 (Muminov 1970, S. 190); die Zahl 134.000 stammt von Sulkevich 1940, S. 50. Die Volkszählung 1939 ergab 136.283, wurden aber von Zhiromskaya (1992) angefochten, indem sie die bewusste Verfälschung der Daten „nach oben " beschreibt und analysiert. Ein weiterer Grund für die Zahl 191.000 kann darin liegen, dass die städtische Bevölkerung des gesamten Gebiets (oblast') gezählt wurde, zu dem neben Samarkand offenbar noch zwei weitere Städte zählten, die in den Abschlusstabellen gleichwohl nicht ausgewiesen werden.

133 Muminov 1970, S. 232. 
zwischen 200.000 und 225.000 Menschen. ${ }^{134}$ Die offiziellen Angaben der Volkszählungen der Jahre 1959 und 1970 berichten von 202.497 bzw. 275.990 Stadtbewohnern. ${ }^{135}$ Selbst wenn die Zahlen nicht sicher sind, so wird immerhin deutlich, dass ein Bevölkerungszuwachs von 60-80.000 Menschen pro Jahrzehnt zu verzeichnen war, was eine enorme Herausforderung für die Stadt dargestellt haben muss.

Die unionsweite Erhebung des Wohnraumbestandes von 1960 zeugt davon, dass viele Bewohner von Samarkand sich in ziemlich beengter Wohnsituation befanden: Die durchschnittliche Wohnfläche pro Person im Jahre 1950 hatte 5,6 Quadratmeter betragen, welche sich in den folgenden zehn Jahren sogar noch auf 5,0 Quadratmeter verschlechtert hatte. ${ }^{136}$ In individuellen Häusern war der durchschnittliche verfügbare Raum mit lediglich 4,8 Quadratmetern pro Person sogar noch geringer.

Bezeichnend ist auch, dass in diesen Berichten fast nie von einem „Haus“ (dom) die Rede ist, sondern von „Bauten“ oder „Wohnbauten“ (stroenie, zhiloe stroenie) und „Wohnungen“ (kvartiry) in diesen Bauten. ${ }^{137}$ Laut den erhobenen Zahlen war die durchschnittliche Größe von so einem Wohnbau im Jahr 1960 38,3 Quadratmeter, während eine Wohnung im Durchschnitt 22,4 Quadratmeter hatte. Der Vergleich zwischen der durchschnittlichen Größe einer Wohnung und eines Zimmers (siehe Tabelle) zeigt, dass die meisten dieser Wohnungen offenbar nur aus einem Zimmer bestanden hatten, während ein Wohnbau im Schnitt aus 1,2 Wohnungen bestand. Die Situation hatte sich dabei im Vergleich zu 1950 nicht wesentlich verbessert, obwohl die absolute Zahl der Wohnbauten und Wohnungen um 10.000 gestiegen war.

134 SamOGA, f. 26, op. 1, d. 1363, 1. 3.

135 Eine digitalisierte Sammlung der Volkszählungen in der Sowjetunion kann unter folgender Web-Adresse abgerufen werden: http://www.demoscope.ru/weekly/ssp/census.php (letzter Zugriff am 16.07.2020).

136 TsGARUz, f. 1619, op. 16, d. 4, 1. 25. Die Angabe bezieht sich auf „Wohnfläche“ dividiert durch Bewohnerzahl. Nimmt man stattdessen die „Gesamtfläche“ (also einschließlich Küche, Flur etc., so ergibt sich die Zahl von 6,5 Quadratmeter pro Person - eine Zahl, die „nach oben“ eher weitergegeben werden konnte.

137 Bei der Übersetzung habe ich mich für „Wohnbau“ und nicht „Wohngebäude“ entschieden, da dies die Konnotationen des russischen Wortes „stroenie“ besser wiedergibt. 
Tabelle: Zahlen aus der Wohnraumbestandszählung in Samarkand (1960) in $\mathrm{m}^{2}:^{138}$

\begin{tabular}{lllllllll}
\hline $\begin{array}{c}\text { Zahl der } \\
\text { Wohn- } \\
\text { bauten }\end{array}$ & $\begin{array}{c}\text { Wohn- } \\
\text { fläche }\end{array}$ & $\begin{array}{c}\text { Gesamte } \\
\text { Fläche }\end{array}$ & $\begin{array}{c}\text { Durchschn. } \\
\text { Größe } \\
\text { eines } \\
\text { Wohnbaus }\end{array}$ & $\begin{array}{c}\text { Gesamt- } \\
\text { zahl der } \\
\text { Wohnun- } \\
\text { gen }\end{array}$ & $\begin{array}{c}\text { Durch- } \\
\text { schn. } \\
\text { Größe } \\
\text { einer Woh- } \\
\text { nung }\end{array}$ & $\begin{array}{c}\text { Zim- } \\
\text { mer } \\
\text { insge- } \\
\text { samt }\end{array}$ & $\begin{array}{c}\text { Durchschn. } \\
\text { Größe } \\
\text { eines } \\
\text { Zimmers }\end{array}$ \\
\hline 1950 & 14.056 & 420.205 & 504.387 & 35,9 & 19.289 & 21,8 & 28.614 & 21,7 \\
1960 & 23.599 & 662.912 & 905.418 & 38,3 & 29.561 & 22,4 & 49.098 & 23,1 \\
\hline
\end{tabular}

Ein Beispiel dafür, wie solche Gebäude ausgesehen haben, kann man zum Teil noch heute in den sogenannten Gemeinschaftshöfen (obshchij dvor) auffinden ein Phänomen, das sich vor allem im russisch-kolonialen Stadtteil von Samarkand herausgebildet hat. Es handelt sich um 80-100 Meter lange Höfe hinter den im 19. Jahrhundert errichteten Vorderhäusern, Höfe, die im Laufe des 20. Jahrhunderts mit Anbauten unterschiedlicher Größe und Massivität gefüllt wurden. ${ }^{139}$ Viele meiner Interviewpartner berichteten vom Leben in solchen Höfen, das dem in einer Kommunalwohnung ähnelte, nur eben nicht in einer Etagenwohnung, sondern - mit einer Toilette vom Typus „Plumpsklo“ - in einem Gemeinschaftshof. ${ }^{140}$

Archivdokumente des städtischen Ispolkom liefern zahlreiche Zeugnisse der schwierigen Wohnsituation in Form von Bittschriften und Anträgen auf Verbesserung, in denen die Lage sehr anschaulich beschrieben wird. So lässt sich einem Beschluss entnehmen, dass eine Frau mit ihrer fünfköpfigen Familie in einem Haus lebte, das lediglich aus einem Zimmer (14 Quadratmeter) und einer Küche (9 Quadratmeter) bestand. ${ }^{141}$ Ein anderer Kläger schildert eine Situation, in der er mit seiner achtköpfigen Familie (einer Frau und sechs Kindern) ein kleines Zimmer bei der Verwandtschaft bewohnt; er bittet daher um eine Parzelle, um sich ein eigenes Haus bauen zu können. ${ }^{142}$

138 TsGARUz, f. 1619, op. 16, d. 5, 1. 58.

$139 \mathrm{Zu}$ der Beschreibung des Phänomens siehe Gangler et al. 2006, S.220-227, sowie Buttino 2015, S. 69 ff.

140 In den Ausführungen hier beziehe ich mich vor allem auf die Viertel, die nach dem Zweiten Weltkrieg entstanden sind. Über das Leben in der Altstadt aus der Perspektive von unterschiedlichen ethnischen Gruppen berichtet Marco Buttino, 2015, insbesondere Kapitel 3 und 4.

141 SamOGA, f. 26, op. 1, d. 2387, 1. 96.

142 SamOGA, f. 26, op. 1, d. 2156, 1. 17. 
Für die städtischen Behörden selbst war die prekäre Situation ebenfalls kein Geheimnis; in den Berichten und Diskussionsprotokollen finden sich recht nüchterne und kritische Lagebeschreibungen. Eines der eindrucksvollsten Beispiele für die Wohnungsnot lieferte mir bei der Archivrecherche eine Anordnung aus dem Jahr 1954 zu Aufräumarbeiten an den historischen Baudenkmälern am Registan-Platz: In einem der Punkte wird angeordnet, Räume wie z.B. die ehemaligen Lernklausen der Medresen von ihren Bewohnern zu „befreien“..143

Laut Statistik hatten im Jahr 1960 22.787 Menschen in Samarkand keinen eigenen, d.h. weder im Eigenbesitz befindlichen noch vom Staat zugeteilten, Wohnraum. Interessanterweise waren aber lediglich 12.000 Personen (2814 Familien) auf einer Warteliste für eine Wohnung registriert. ${ }^{144}$ Eine direkte Erklärung hierfür geben die Quellen nicht; eine naheliegende Vermutung wäre, dass die restlichen Zehntausend offenbar zu wenig Chancen sahen, ihre Wohnungssituation auf diesem Wege zu verbessern.

Die statistischen Berichte geben auch Auskunft über das Verhältnis zwischen individuellem und vergemeinschaftetem (obobshchestvlennyi ${ }^{145}$ ) Wohnraumbestand. Berechnet an den vorhandenen Zahlen, machte der individuelle Sektor von Samarkand mit 905.418 Quadratmetern 63 Prozent des gesamten Wohnraumbestandes aus. Damit lag Samarkand sogar unter dem Durchschnitt der Republik - im Jahr 1960 waren 66,6 Prozent der Wohnungen in Usbekistan als individuelles Eigentum registriert. ${ }^{146}$

Das unionsweite Wohnungsbauprogramm, das 1957 ansetzte, sollte die Situation entschärfen, sodass mehr Menschen sich selbst eigenen Wohnraum bauen oder welchen vom Staat bekommen konnten. Doch die Kapazitäten reichten nicht aus, um die Wohnungsnot ein für allemal zu beseitigen. Ein Planungsdokument des städtischen Ispolkom aus dem Jahr 1970 zu vorgesehenen Umsiedlungen von Menschen aus baufälligen Wohnungen, Baracken, Halbkeller-Wohnungen und ähnlichem beklagenswerten Wohnraum spricht von über 2000 Personen (676 Familien), die bis 1975 umgesiedelt werden sollten. ${ }^{147}$

143 SamOGA, f. 26, op. 1, d. 1673, 1. 35 und f. 1617, op. 1, d. 40, 1. 72-74.

144 SamOGA, f. 26, op. 1, d. 2168, 1. 170.

145 Die Bezeichnung bezieht sich auf zwei der Besitzformen des Wohnraums, und zwar Wohnraum im Besitz der lokalen Sowjets beziehungsweise der Betriebe und anderer Einrichtungen.

146 TsGARUz, f. 1619, op. 16, d. 4, 1. 33-34.

147 SamOGA, f. 1617, op. 1, d. 218, 1. 23-24. 


\section{Wohn- und Stadtinfrastruktur}

Nach dem Zweiten Weltkrieg funktionierte Samarkand noch weitgehend auf Basis der Infrastruktur aus der Vorkriegszeit. Zeitgenössische Berichte der städtischen Behörden vermitteln zusammen mit den Erinnerungen von Zeitzeugen ein recht desolates Bild. Diese Situation war aber nicht singulär oder spezifisch für Samarkand, vielmehr gehörten infrastrukturelle Mängel in zahlreichen Städten der Sowjetunion zu Beginn der 1950er-Jahre zur Normalität. ${ }^{148}$ So schlug sich eben auch für Samarkand die allgemeine Ressourcenknappheit der Nachkriegsjahre in der städtischen Wirtschaft nieder.

Maroder und baufälliger kommunaler Wohnraumbestand ist ein häufiges Thema der Berichte der städtischen Wirtschaftskommission und des Stadt-Ispolkom, die sich über die Nichterfüllung der Instandsetzungspläne beklagen. ${ }^{149}$ So ist z.B. für das Jahr 1950 die Rede von 120 baufälligen Bauten, bei deren Reparatur nicht nur das Dach, sondern auch Wände und teilweise die Fundamente erneuert werden mussten. ${ }^{150}$ Im Jahr 1954 fügten regenreiche Winter- und Frühlingsmonate der ohnehin angeschlagenen Bausubstanz erheblichen Schaden zu: 521 Bauten zählten als baufällig, 71 als komplett zerstört, die Bewohnerschaft wurde „verdichtet“ oder in Kellerräumen untergebracht. ${ }^{151}$ Das meistverbreitete Baumaterial war Lehm bzw. ungebrannte Lehmziegel, was die Häuser besonders anfällig und reparaturbedürftig machte.

Bei aller Notwendigkeit, die Häuser zu reparieren bzw. wieder bewohnbar zu machen, fehlten den Behörden häufig Arbeitskräfte und Baumaterial. Das Büro für Instandhaltung und Reparatur beklagt jahrein, jahraus immer wieder die schlechte Ausstattung und den Mangel an Baumaterialien; so war z.B. das Fehlen von Bauholz ein Leitmotiv zahlreicher Schreiben und Berichte des Jahres 1954. ${ }^{152}$ In einem der Sitzungsprotokolle wird beklagt, dass das Reparaturbüro an technischen Mitteln lediglich über „zwei Autos und eine Kreissäge“ verfüge. ${ }^{153}$ Zusätzlich werden bei den unterschiedlichen verantwortlichen Stellen auch hauseigene Missstände wie schlechte Organisation, mangelnde Arbeitsdisziplin und Mittelveruntreuung angeprangert. ${ }^{154}$ Klagen, Vorwürfe und Appelle an die Schuldigen

\footnotetext{
148 Bohn 2008, S. 257.

149 SamOGA, f. 26, op. 1, d. 1427, 1. 2.

150 SamOGA, f. 26, op. 1, d. 1363, 1. 3.

151 SamOGA, f. 26, op. 1, d. 1667, 1. 40-42.

152 SamOGA, f. 26, op. 1, d. 1667, 11. 123, 127.

153 SamOGA, f. 26, op. 1, d. 1437, 1. 68-69.

154 SamOGA, f. 26, op. 1, d. 1398, 1. 66.
} 
sind unter den häufigsten Themen der Berichte und Sitzungsprotokolle der kommunalen Behörden, die ich im Stadtarchiv finden konnte.

Probleme gab es auch in der Wasserversorgung. Ein Bericht aus dem Jahr 1951 gibt Auskunft: Demnach benutzten die städtischen Wasserwerke noch größtenteils die Infrastruktur aus dem Jahr 1933. Sie waren damit imstande, dem System 7.000 Kubikmeter Wasser pro Tag zuzuführen, während der Bedarf aber 20.000 Kubikmeter pro Tag betrug. Dabei verbrauchten industrielle Betriebe 60 Prozent des verfügbaren Wassers, während mehr als die Hälfte der Bevölkerung Wasser aus Brunnen und aryks (Bewässerungsgräben) nutzte. ${ }^{155}$ Ähnliche Erinnerungen habe ich auch in Gesprächen mit Zeitzeugen erzählt bekommen: Das Wasser (auch zum Trinken!) stammte aus den aryks, die allerdings früher sauberer gewesen sein sollen. „In ein aryk zu spucken war ein Unding“, so einer der Interviewpartner. Bestenfalls kam das Wasser aus einer öffentlichen Wasserpumpe, von denen es in der Stadt im Jahre 1963 noch 465 Stück gab. ${ }^{156}$

Am schnellsten ging der Ausbau des Stromnetzes voran: Obwohl 29 Prozent des Wohnraumbestandes im Jahre 1950 noch recht mangelhaft versorgt waren, betrug die Elektrifizierung im Jahr 1960 bereits 83 Prozent. ${ }^{157}$ Die Situation mit Wasserver- und Abwasserentsorgung sah viel schlechter aus: Vom Wohnraum in individuellem Besitz waren 1950 lediglich 1,4 Prozent mit Wasserleitungen versorgt, bis 1960 stieg diese Zahl auf 4,0 Prozent. Beim Wohnraum in öffentlicher Hand stieg die Versorgungsrate innerhalb des gleichen Jahrzehnts von 3,7 auf 10,2 Prozent. ${ }^{158}$ Der Bau der Kanalisation wurde den Archivquellen zufolge erst 1958 begonnen, aber auch die Benutzung von konventionellen Sickergruben war nicht immer unproblematisch, denn bei der Sanitärbrigade fehlten Saugwagen: Im Jahr 1951 hatte sie nur zehn von den siebzehn übrig, die die Stadt vor dem Krieg besaß. ${ }^{159}$ Die kommunale Infrastruktur litt auch an Problemen mit der Müllabfuhr und der Straßen- und aryk-Reinigung.

In den 1960er-Jahren, insbesondere in deren zweiter Hälfte, flossen mit der Intensivierung der industriellen Bauweise und der Errichtung neuer Viertel mit

155 SamOGA, f. 26, op. 1, d. 1437, 1. 107.

156 SamOGA, f. 26, op. 1, d. 2168, 1. 36.

157 TsGARUz, f. 1619, op. 16, d. 4, l. In den Statistiken lassen sich die Angaben nur für individuelle Bauten finden. Aus Gründen der Einfachheit übernehme ich diese auch für den staatlichen Wohnraumbestand, es ist zu vermuten, dass dieser sogar etwas höher war.

158 TsGARUz, f. 1619, op. 16, d. 4, 1. 26, 29.

159 SamOGA, f. 26, op. 1, d. 1397, 1. 37. Dieses Problem hielt offenbar sehr lange an. So erinnerte sich einer der Interviewpartner, dass er noch in den 1980er-Jahren mehrere Monate auf einen Saugwagen warten musste, der zu alledem am Ende nur ein Fünftel der Fäkalien mitnehmen konnte. 
Etagenbauten, die eine bestimmte Infrastruktur verlangten, offenbar auch mehr Mittel in den infrastrukturellen Ausbau. Dies wird anhand steigender Zahlen deutlich. ${ }^{160}$ Die Stadt bzw. einige Bezirke profitierten von Wohnungsbauprogramm und Industrialisierung, obwohl das schnelle Wachstum auch deutliche Probleme mit sich brachte. In einem Schriftstück aus dem Jahr 1963/64, einer Art Steckbrief für das Gebiet von Samarkand, werden in Stadt und Umland zahlreiche städtebauliche Mängel diagnostiziert, darunter beispielsweise eine gegen den Generalplan verstoßende falsche Platzierung von Industriebetrieben (z.B. zu nah an Wohngebieten) und deren negative Auswirkungen auf die sanitäre Situation. Dazu kam die Überlastung der schlecht entwickelten Wasser- und Abwassersysteme. „Fast alle Wasserarterien der Städte sind verseucht/verdreckt, insbesondere in Samarkand, da häufig die Kanalisation fehlt und zahlreiche Industriebetriebe noch keine eigene Kläranlage haben“, heißt es weiter in dem Text. Des Weiteren wird die mangelhafte Versorgung der Stadt mit öffentlichen Grünanlagen kritisiert; diese betrug lediglich 0,61 Quadratmeter bei einer Norm von 16 Quadratmetern pro Person. Insgesamt werden sowohl der technischen Dokumentation als auch der Bauqualität große und teilweise schwerwiegende Mängel attestiert. Außerdem wird in dem Dokument über ein großes Problem der städtischen Baubehörden gesprochen, das hier später intensiv besprochen und diskutiert wird - nämlich über einen Landkonflikt der Stadt mit den umliegenden Kolchosen. ${ }^{161}$

Die Lage in Samarkand ist hier nur ein Beispiel für die Überforderung des sowjetischen Staates bei der Umsetzung eigener sehr hoch gesteckter Modernisierungsvorhaben und die Überlastung der lokalen Verwaltung, die beim Ausbau der bereits vor dem Krieg unzureichend gewesenen Infrastruktur dem Bedarf weit hinterherhinkte. In der Wohnungsfrage entwickelten sowohl die Bürger als auch die Verwaltung diverse Strategien, um ihre jeweiligen Ziele zu erreichen. Einen kurzen Überblick darüber bietet der nächste Abschnitt.

160 Mumiov 1970, Bd. 2, S. 410.

161 SamOGA, f. 1617, op. 1, d. 153, 1. 1-2. Wie bei vielen der gefundenen Dokumente lässt sich auch hier die Reaktion des Ministeriums nicht feststellen. 


\section{Staatliche und individuelle Strategien zur Beseitigung des Wohnraummangels}

Die Last der Investitionen für den Wohnungsbau wurde in den Städten der Sowjetunion auf mehrere Schultern verteilt. Die vorliegende Studie befasst sich ausführlich mit dem individuellen Wohnungsbau, doch zuvor möchte ich eine kurze Übersicht über weitere Möglichkeiten geben.

Der staatliche Sektor umfasste neben dem kommunalen auch den sogenannten ,betrieblichen“ Wohnungsbau, vedomstvennoe zhilishchnoe stroitel'stvo: Betriebe, diverse Einrichtungen und Organisationen waren angehalten, ihre Mitarbeiter mit Wohnraum zu versorgen. Dazu sollten sie teils aus eigenen Mitteln, teils durch erstrittene Zuwendungen (je nach Sparte unterstanden sie unterschiedlichen Ministerien und konnten auf diesem Wege Extrafinanzierungen für Bauprojekte erhalten) Wohnraum für ihre Beschäftigten bauen. ${ }^{162}$ Für die Betriebe stellte diese Aufgabe einen zusätzlichen Aufwand dar, während der Erfolg vom Wichtigkeitsgrad der jeweiligen Organisation und der Initiative und Durchsetzungsfähigkeit ihrer Leitung abhing. Das folgende Zitat aus einem Sitzungsprotokoll des städtischen Ispolkom illustriert die Forderungen, mit denen die Betriebsleitungen konfrontiert wurden, und erklärt zugleich deren Situation:

[...] nicht nachvollziehbar bleibt das Verhalten derjenigen Leiter von Betrieben, die keine energischen Versuche unternehmen, Zuwendungen für den Wohnungsbau zu erstreiten, und sich keine zusätzlichen Schwierigkeiten zum Wohle der besseren Befriedigung der Bedürfnisse der Arbeiter auferlegen. ${ }^{163}$

Dies war nur einer der Gründe für die Nichterfüllung der angesetzten Pläne für den Bau neuer Wohnungen, welche selbst bereits nicht ausreichend waren, um den Bedarf an Wohnraum zu decken. Über das mangelhafte Engagement der Betriebe beklagt sich der Stadt-Ispolkom auch in einem Schreiben an den Ministerrat der UzSSR. Dieses Schreiben enthält außerdem die Bitte, der Wohnungsbau mehrerer Betriebe im Umfang von insgesamt 210 Wohnungen möge doch in das Plansoll des Jahres 1954 eingeschlossen werden. ${ }^{164}$ Wenn wir uns in Erinnerung rufen, dass im Jahr 1960 (also sechs Jahre später) 2800 Familien auf einer Warteliste standen, wird deutlich, wie unzureichend diese Baumaßnahmen waren. Hinzu kamen die schlechte Finanzierung, Knappheit an Baumaterialien und qua-

162 Mündliche Auskunft: Architekt O.P. aus Samarkand.

163 SamOGA, f. 26, op. 1, d. 1933, 1. 12. Übersetzung der Autorin.

164 SamOGA, f. 26, op. 1, d. 1667, 1. 21-22. 
lifizierter Arbeitskraft, „nicht zufriedenstellende Arbeitsdisziplin“ und die bürokratischen Hürden des sowjetischen Planungssystems. Ähnliche prinzipielle Probleme in der staatlichen Bauorganisation und Bürokratie beschreibt bereits Alfred John DiMaio. ${ }^{165}$

Die politischen und finanziellen Voraussetzungen für eine ernsthafte quantitative Steigerung wurden erst nach der Ankündigung des Wohnungsbauprogramms im Jahr 1957 geschaffen. Mit Hilfe von industrieller Bauweise sollten Wohnhäuser schneller und kostengünstiger errichtet werden können. Um dies auch technisch durchführbar zu machen, musste erst die Baumaterialindustrie so weit ausgebaut werden, dass sie dem gesteigerten Bedarf gerecht werden konnte; vor Ort mussten spezielle Baukombinate, sogenannte DSK (domostroitel'nye kombinaty), errichtet werden, was zugleich das Problem der sonst sehr hohen Transportkosten lösen sollte. Den Archivquellen und Gesprächspartnern zufolge wurde der Bau des ersten Kombinats in Samarkand gegen 1958 abgeschlossen, während die tatsächliche Fertigstellung der ersten Wohnhäuser erst für 1962 verbürgt ist. ${ }^{166}$ Auf der bürokratischen Ebene wurden mit dem Programm die Städte bzw. die Ispolkom zu den Hauptauftraggebern des Wohnungsbaus gemacht, was die Prozesse zentralisieren sollte.

Wie bereits oben beschrieben, setzte der Staat für die Linderung des Wohnraummangels parallel auf eine zweite Säule - auf den individuell finanzierten Wohnungsbau in Form von eigenen Häusern, und ab 1962 zusätzlich auf Kooperativen. Genauer gesagt handelte es sich bei beiden Formen um eine Mischfinanzierung, denn im individuellen Wohnungsbau unterstützte der Staat die Bürger durch Kredite und Landzuteilung; beim kooperativen Wohnungsbau wiederum, in dessen Zuge Etagenbauten entstehen sollten, sollten die Bürger fast die komplette Finanzierung des Bauvorhabens selbst übernehmen. ${ }^{167}$

Die Zielpersonen dieser staatlichen Maßnahmen verfügten ihrerseits über unterschiedliche Ressourcen. Dazu zählten sowohl finanzielle Mittel als auch handwerkliche Fertigkeiten bzw. die Fähigkeit, sich Arbeitskräfte zu mobilisieren, ein bestimmter Bildungsgrad und die Fähigkeit, sich das sowjetische bürokratische System nutzbar zu machen. Die Optionen und Möglichkeiten, die persönliche Wohnungsnot mit Hilfe eines eigenen Hauses zu lösen, reichten von offizieller Antragstellung mit langen Wartezeiten bis zum Kauf eines fertigen

165 DiMaio 1974, S. 88 ff.

166 Die Mängel wurden sowohl in den Berichten der Behörden festgehalten und kritisiert als auch in der offiziellen „Geschichte von Samarkand“ bei Muminov 1970.

167 Ausführlicher über den sowjetischen kooperativen Wohnungsbau siehe z.B. DiMaio 1974, S. 23 ff.; Harris 2013, S. 154 ff. 
Hauses. Diese sind später ausführlich zu beschreiben und werden daher hier nicht weiter ausgeführt. Denjenigen Menschen aber, für die der Bau eines Hauses aus eigener Kraft samt Investition von Geld und Arbeit keine Option darstellte, blieb nur die Möglichkeit, sich auf eine Warteliste für die Zuteilung von kommunalem Wohnraum setzen zu lassen. Je nach Beschäftigungsart geschah dies direkt bei der Stadt, im Betrieb oder - je nach Gesetzgebung - beim Bezirks- oder Stadt-Ispolkom. Hier bestand ebenfalls keine Garantie, und das Warten konnte Jahre dauern, während zahlreiche Menschen zur Miete oder bei Verwandten in häufig sehr beengten Verhältnissen lebten. Grad der Bedürftigkeit, Durchsetzungsfähigkeit, Privilegien und/oder Kontakte konnten die Wartezeit auf der Liste verkürzen.

Eine weitere Möglichkeit, sich selbst zu helfen, wenn man glaubte, von den lokalen Behörden im Stich gelassen zu sein, war, sich an höhere Instanzen zu wenden. Dies scheint eine recht verbreitete Praxis gewesen zu sein: In einem Beschluss des Stadtrates von Samarkand ist die Rede von 449 Beschwerden, die die Behörde aus verschiedenen höheren Parteiorganisationen oder Instanzen erreicht hatten. ${ }^{168}$ Zwei meiner Gesprächspartner berichteten davon, selbst mit Hilfe solch einer Beschwerde nach jahrelangem Warten letztlich doch eine Wohnung bekommen zu haben. Bezeichnend ist, dass beide Interviewten unabhängig voneinander betonten, wie wichtig die Kompetenz (die eigene bzw. die der Eltern) war, sich an die richtige Instanz zu wenden, die im Idealfall auf Unionsebene lag, da Beschwerden auf Republikebene häufig wirkungslos blieben.

168 SamOGA, f. 1658, op. 2, d. 139, 1. 127. 